PAPER • OPEN ACCESS

\title{
A computationally efficient method for the attenuation of alternating current stimulation artifacts in electroencephalographic recordings
}

To cite this article: Roberto Guarnieri et al 2020 J. Neural Eng. 17046038

View the article online for updates and enhancements.

The Department of Bioengineering at the University of Pittsburgh Swanson School of Engineering invites applications from accomplished individuals with a $\mathrm{PhD}$ or equivalent degree in bioengineering, biomedical engineering, or closely related disciplines for an open-rank, tenured/tenure-stream faculty position. We wish to recruit an individual with strong research accomplishments in Translational Bioengineering (i.e., leveraging basic science and engineering knowledge to develop innovative, translatable solutions impacting clinical practice and healthcare), with preference given to research focus on neuro-technologies, imaging, cardiovascular devices, and biomimetic and biorobotic design. It is expected that this individual will complement our current strengths in biomechanics, bioimaging, molecular, cellular, and systems engineering, medical product engineering, neural engineering, and tissue engineering and regenerative medicine. In addition, candidates must be committed to contributing to high quality education of a diverse student body at both the undergraduate and graduate levels. 


\title{
Journal of Neural Engineering
}

PAPER

CrossMark

RECEIVED
7 April 2020
REVISED
26 June 2020
ACCEPTED FOR PUBLICATION
27 July 2020
PUBLISHED
17 August 2020

\section{A computationally efficient method for the attenuation of alternating current stimulation artifacts in electroencephalographic recordings}

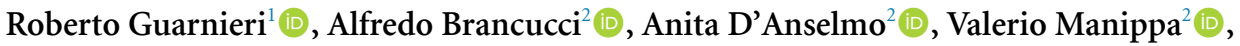 \\ Stephan P Swinnen ${ }^{1,3}$ (D) Franca Tecchio ${ }^{4}$ (D) and Dante Mantini ${ }^{1,5,6}$ (D) \\ 1 Research Center for Motor Control and Neuroplasticity, KU Leuven, Tervuursevest 101, 3001, Leuven, Belgium \\ 2 Department of Psychological, Health and Territory Sciences, "G. d'Annunzio" University of Chieti-Pescara, Chieti 66100, Italy \\ 3 Leuven Brain Institute, KU Leuven, 3000, Leuven, Belgium \\ 4 Laboratory of Electrophysiology for Translational Neuroscience, Institute of Cognitive Sciences and Technologies, National Research \\ Council (CNR), 00185, Rome, Italy \\ 5 Brain Imaging and Neural Dynamics Research Group, IRCCS San Camillo Hospital, 30126, Venice, Italy \\ ${ }^{6}$ Author to whom any correspondence should be addressed. \\ E-mail: dante.mantini@kuleuven.be
}

Keywords transcranial alternating current stimulation (tACS), closed-loop, low-computational processing, artifact removal, electroencephalography (EEG), principal component analysis (PCA)

\begin{abstract}
Objective. Recent studies suggest that the use of noninvasive closed-loop neuromodulation combining electroencephalography (EEG) and transcranial alternating current stimulation (tACS) may be a promising avenue for the treatment of neurological disorders. However, the attenuation of tACS artifacts in EEG data is particularly challenging, and computationally efficient methods are needed to enable closed-loop neuromodulation experiments. Here we introduce an original method to address this methodological issue. Approach. Our alternating current regression (AC-REG) method is an adaptive (time-varying) spatial filtering method. It relies on a data buffer of preset size, on which principal component analysis (PCA) is applied. The resulting components are used to build a spatial filter capable of regressing periodic signals in phase with the stimulation. PCA is performed each time that a new sample enters the buffer, such that the spatial filter can be continuously updated and applied to the EEG data. Main results. The AC-REG accuracy in terms of tACS artifact attenuation was assessed using simulated and real EEG data. Alternative offline processing techniques, such as the superimposition of moving averages (SMA) and the Helfrich method (HeM), were used as benchmark. Using simulations, we found that AC-REG can yield a more reliable reconstruction of the stimulation signal for any frequency between 1 and $80 \mathrm{~Hz}$. Analysis of real EEG data of 18 healthy volunteers showed that AC-REG was able to better recover hidden neural activity as compared to SMA and HeM. Also, significantly higher correlations between power spectrum densities in tACS on and off conditions, respectively, were obtained using AC-REG $(r=0.90)$ than using SMA $(r=0.80)$ and HeM $(r=0.86)$. Significance. Thanks to its low computational complexity, the AC-REG method can be employed in noninvasive closed-loop neuromodulation experiments, with potential applications both in healthy individuals and in neurological patients.
\end{abstract}

\section{Introduction}

During closed-loop neuromodulation experiments, neural activity is continuously recorded, relevant signal signatures are extracted, and the applied magnetic/electrical stimulation is dynamically adapted based on those features (Rebesco et al 2010,
Guggenmos et al 2013). Notably, closed-loop neuromodulation has been shown to facilitate and/or enhance neural plasticity processes as compared to open-loop neuromodulation, for which a fixed set of stimulation parameters is chosen a-priori by the experimenter (Sun and Morrell 2014). The adaptive nature of closed-loop neuromodulation intrinsically 
leads to a lower intra- and inter-subject variability (Iturrate et al 2018), opening the way to its use for the treatment of neurological and psychiatric disorders (Sun and Morrell 2014). Probably, one of the most promising applications of closed-loop neuromodulation is based on deep brain stimulation (DBS) of the basal ganglia for the treatment of movement disorders, including Parkinson's disease, essential tremor and dystonia (Perlmutter and Mink 2006). DBS is however invasive, and not suitable for a wide range of other conditions involving malfunctioning of large-scale brain networks rather than very specific brain regions.

Transcranial alternating current stimulation (tACS) is a non-invasive neuromodulation approach that can be suitable for use in a wider number of neurological and psychiatric conditions (Woods et al 2016). With tACS, small amounts of current are injected into the scalp via rubber electrodes enclosed in saline soaked sponges (Morales-Quezada et al 2014). The spatial specificity of the stimulated area is limited, but the use of multi-channel montages (Alekseichuk et al 2019) and frequency-based interference techniques (Grossman et al 2017) can mitigate this potential problem. The number of studies employing tACS for addressing basic and clinical neuroscience questions is nowadays very large (Alexander et al 2019, Dowsett et al 2019). There is considerable experimental (Marshall et al 2011, Herrmann et al 2013) and computational (Ali et al 2013, Merlet et al 2013) evidence that tACS can effectively entrain brain oscillations. It has been successfully used to modulate vision (Vossen et al 2015), movement (Feurra et al 2011) and audition (Riecke et al 2015). Moreover, functional magnetic resonance imaging showed that tACS can induce short-term neuroplastic effects over relatively specific cortical regions (Cabral-Calderin et al 2016, Bächinger et al 2017).

When using tACS for closed-loop neuromodulation, neural signals usually need to be acquired in a noninvasive manner, for instance using electroencephalography (EEG). EEG electrodes, which are placed over the scalp of the participant, measure the potentials induced by electrical activity of pyramidal neurons in the cortex (Beres 2017). EEG systems are typically portable and not particularly expensive, especially when the number of recording channels is low. It should be noted, however, that the direct estimate of neural activity in the cortex requires the use of high-density EEG montages, with more than 100 electrodes (Tucker 1993). The combination of EEG and tACS is technically challenging, because of the massive artifact that is mixed in the EEG data, in the form of a quasi-sinusoidal signal with main harmonic at the stimulation frequency. For this reason, most EEG-tACS studies have been so far conducted using an interleaved stimulation protocol, and in particular analyzing the EEG data collected in the offstimulation period (Vossen et al 2015, Mansouri et al 2017, Pahor and Jaušovec 2018).

To the best of our knowledge, few solutions exist to attenuate the tACS artifact from EEG recordings. A first approach that has been proposed is the subtraction of a constant sine wave fitted to the EEG signal. Due to variations in the EEG signal primarily induced by slow changes in electrode conductance and by movements of the participant's head, this solution often yields unsatisfactory results (Helfrich et al 2014). Other tACS methods require the whole EEG recording to be available, and are therefore unsuitable for closed-loop neuromodulation studies (Helfrich et al 2014, Kohli and Casson 2020). For instance, the one proposed by Helfrich and colleagues follows a two-step procedure: an artifact template is first subtracted from the data, and the remaining artifacts are then attenuated using principal component analysis (PCA) (Helfrich et al 2014). Only one method, the superimposition of moving averages (SMA) (Kohli and Casson 2015), has been used for real-time removal of tACS artifacts. However, in its current implementation, SMA can simultaneously process only few EEG signals. Furthermore, it tends to strongly suppress the harmonics associated with the tACS artifact, possibly inducing large distortions in the frequency characteristics of neural signals (Kohli and Casson 2019).

Recent studies suggest that the use of closedloop neuromodulation based on tACS and EEG may be a promising avenue for the treatment of neurological disorders (Semprini et al 2018). Therefore, there is a compelling need of a technological solution with low computational complexity for tACS artifact removal, which could be effectively used with low-density as well as high-density EEG recordings. In this paper we introduce a novel solution to this problem. Our alternating current regression (ACREG) method can be conceptualized as an adaptive (time-varying) spatial filter method. Specifically, we use a data buffer of preset size, on which we apply PCA; the resulting principal components (PCs) are used to build a spatial filter for the regression of periodic signals in phase with the stimulation. PCA is performed each time that a new sample enters the buffer, such that the spatial filter can be continuously updated and applied to the EEG data. In this study we assess the performance of AC-REG using both simulated and real EEG signals. Simulated data are used to define the main parameters of the method and to assess its performance under controlled conditions. A comparison in terms of accuracy with alternative methods is conducted, using both real and simulated data. 

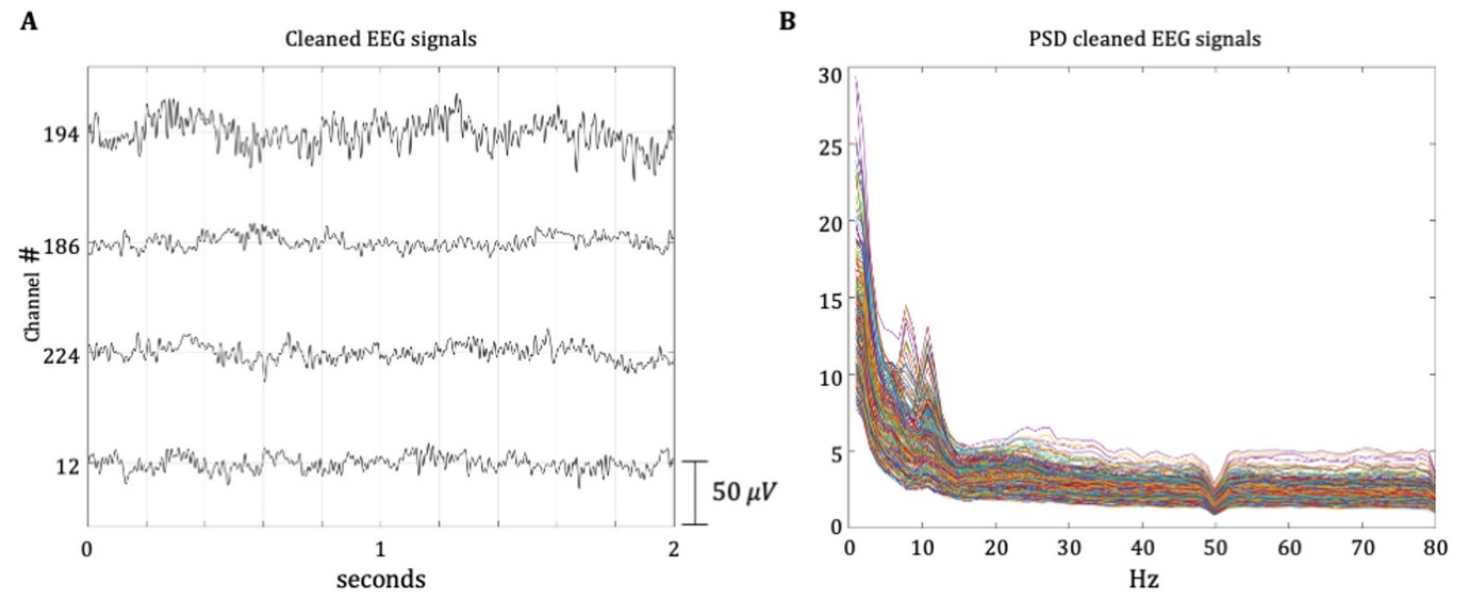

C

D
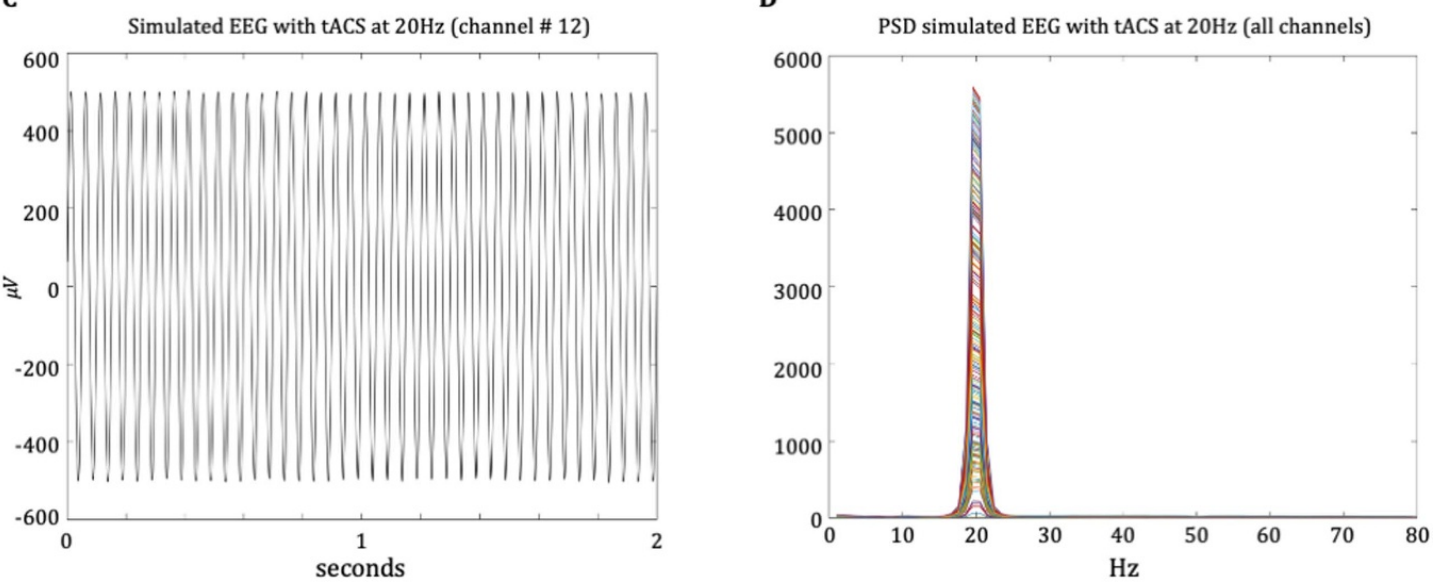

Figure 1. Example of simulated EEG data with tACS artifact. (A) 2-second segment of reconstructed artifact-free EEG signals for four representative channels; (B) power spectral density of the reconstructed artifact-free EEG signals (C) 2-second segment of simulated EEG signals, obtained adding the tACS artifact to the artifact-free EEG signals. (D) power spectral density of the simulated EEG signals with tACS artifact.

\section{Methods}

\subsection{Description of the method}

The AC-REG method relies on the use of EEG signals that are continuously read and stored in a buffer of $n$ samples. A spatial filter is dynamically updated and applied to the most recent data samples to attenuate the contribution of the tACS artifact mixed in the EEG data. For each given time epoch, PCA is calculated on the data buffer to retrieve the PCs, i.e. underlying signals that are statistically uncorrelated with each other and linearly mixed in the data (Turnip and Junaidi 2014). Notably, the number of samples $n$ in the data buffer will inherently have an impact on PCA performance, in terms of accuracy and computation time (Guarnieri et al 2018). The PCA model can be mathematically described as

$$
X(\tau)=A \cdot Y(\tau)
$$

where $X(\tau)$ is a $[k \cdot n]$-dimensional matrix of $k$ recordings and $Y(\tau)$ is a $[k \cdot n]$-dimensional matrix of PC time-courses. Furthermore, $A$ is a $[k \cdot k]$ dimensional matrix that contains the $\mathrm{PC}$ weights over the recordings. The matrices $A$ and $Y(\tau)$ can be estimated using a singular value decomposition (SVD), which imposes a constraint of orthogonality among the PCs (Golub and Kahan 1965, Turnip and Junaidi 2014). After PCA decomposition, the PCs with quasisinusoidal time-courses in phase with the stimulation signal are selected, by imposing a stringent criterion in terms of temporal correlation $(r>0.5)$. This classification of PCs is used to build the spatial filter capable of attenuating tACS artifacts. To this end, a $[n \cdot n]$ diagonal matrix $Z$ is created, setting each element $z_{i i}$ equal to 0 if the $\mathrm{i}$-th PC is classified as related to tACS artifact, or equal to 1 otherwise. The spatial filter $W(t)$ at any given time $\tau$ of the EEG recording, is then obtained as follows:

$$
W(t)=A \cdot Z \cdot A^{-1} .
$$

The vector of EEG data corresponding to the last sample in the buffer, indicated as $X(t)$, is combined with the spatial filter $W(t)$ calculated using the buffer data to obtain the vector of artifact-free EEG $\operatorname{data} X_{c}(t)$ :

$$
X_{c}(t)=W(t) \cdot X(t)
$$




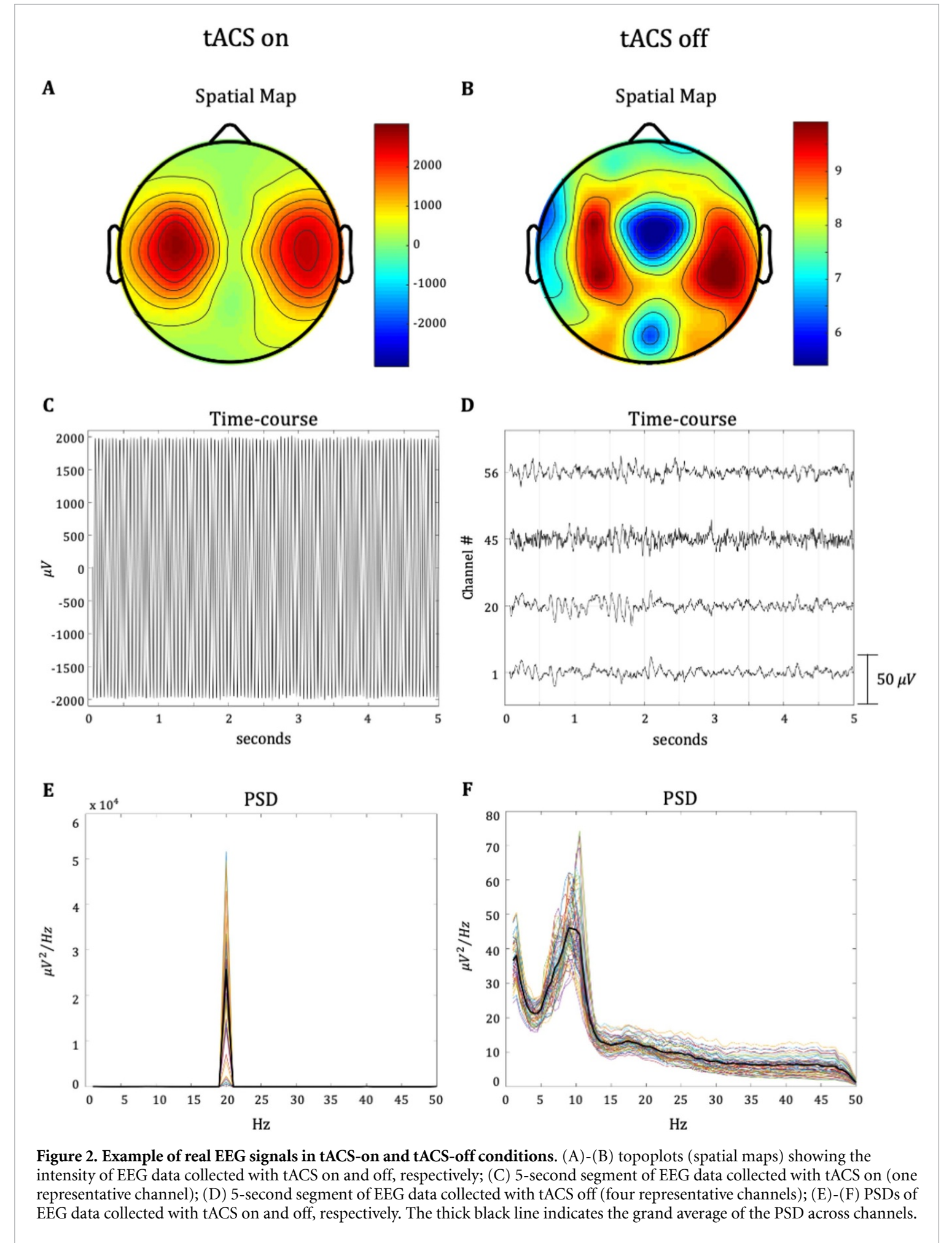

In this manner, the artifacts are subtracted from the EEG recordings with appropriate weights for each channel, and the reconstruction of artifact-free EEG signals is accomplished in a continuous manner.

\subsection{Validation of the method}

2.2.1. Performance analysis using simulated EEG data Simulated data used in this study were derived from resting-state EEG recordings collected for $5 \mathrm{~min}$ in 12 right-handed healthy participants (four males and eight females, with ages ranging from 21 to 43 years). These recordings were already used in some of our previous studies (Liu et al 2017, Guarnieri et al 2018, Samogin et al 2019). Before undergoing the examination, the participants gave written informed consent to the experimental procedures, which were approved by the Institutional Ethics Committee of ETH Zurich. EEG data were recorded at $1000 \mathrm{~Hz}$ using a 256channel system from Electrical Geodesics (Eugene, US). The electrode at vertex (labeled as Cz in the 10/20 international system) was used as physical reference. 

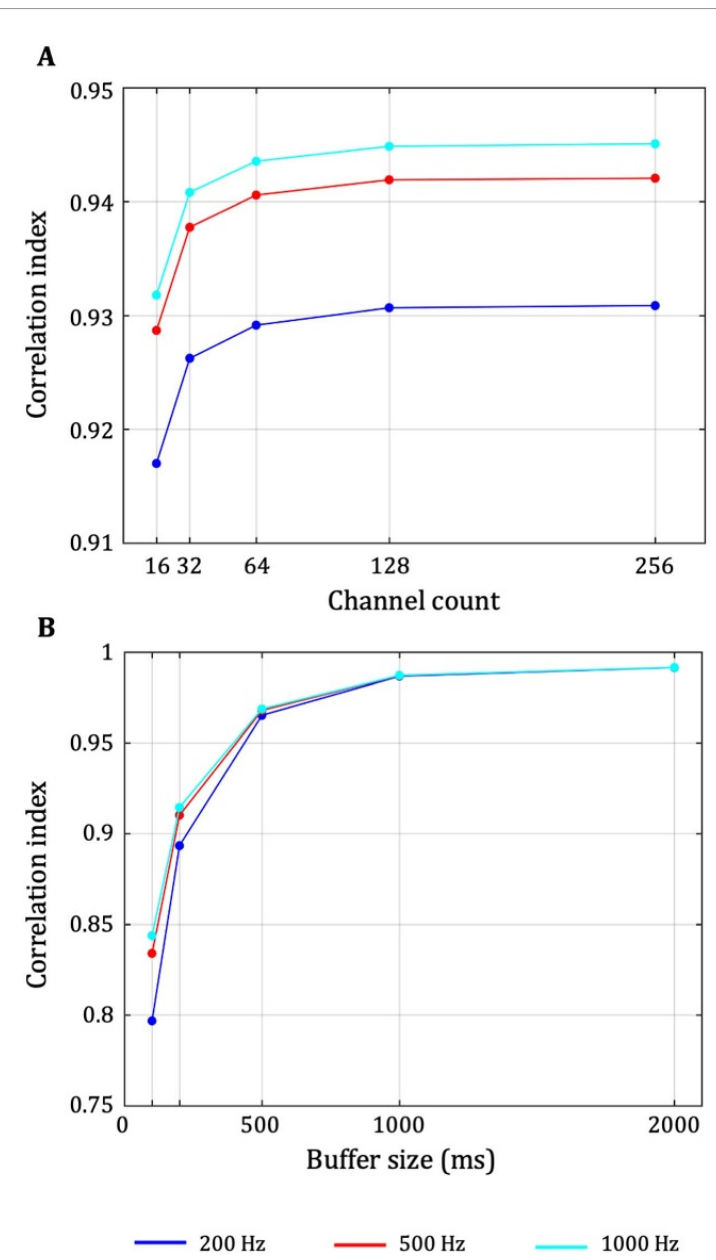

Figure 3. Dependence of AC-REG reconstruction accuracy on channel count, sampling frequency and buffer size. We examined how the accuracy in the reconstruction of the tACS artifact, quantified by the correlation index, is influenced by (A) the number of EEG channels given as input to AC-REG and (B) the number of samples in the data buffer used. The accuracy for different sampling frequencies is shown using lines with different colors.

EEG data preprocessing was carried out using EEGLAB (https://sccn.ucsd.edu/eeglab/index.php) and built-in MATLAB functions. The preprocessing steps included: bad-channel detection and interpolation, digital filtering in the band $1-80 \mathrm{~Hz}$, resampling to $200 \mathrm{~Hz}$ and average re-referencing. In addition, removal of biological artifacts was performed by means of independent component analysis (ICA), as described in Liu et al (2017). The resulting artifact-cleaned EEG data were then mixed with simulated tACS artifact, generated using the open source toolbox ARtACS (https://github.com/agricolab/ARtACS). Temporal changes in artifact amplitude over time were modelled using an Ornstein-Uhlenbeck process. The intensity of the tACS was set as being 100 times larger than background EEG activity, as observed in real data. Simulated tACS artifacts were generated at varying frequencies ranging between 1 and $70 \mathrm{~Hz}$. More detailed analyses were conducted with simulated tACS artifacts at 10,20 and $70 \mathrm{~Hz}$, as

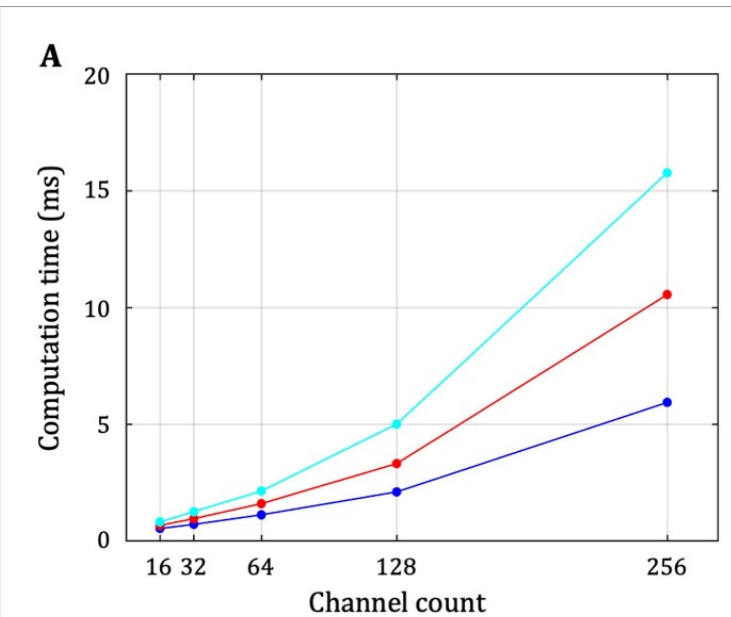

B

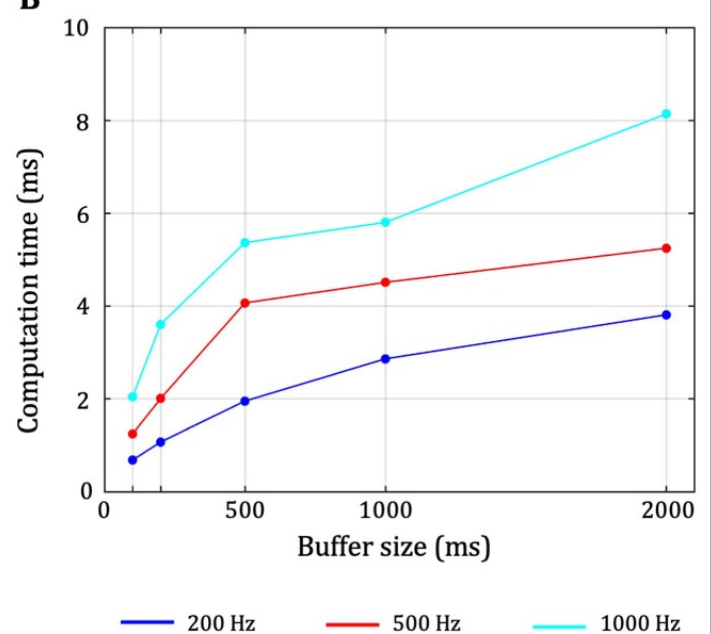

Figure 4. Dependence of AC-REG computation time on channel count, sampling frequency and buffer size. We examined how the computation time is influenced by (A) the number of EEG channels given as input to AC-REG and (B) the number of samples in the data buffer used. The computation time for different sampling frequencies is shown using lines with different colors.

these are frequencies commonly used in tACS studies (Cappon et al 2016, Clayton et al 2018, Sugata et al 2018).

Using the simulated EEG data (see figure 1), we first performed an optimization of AC-REG settings. Pseudo-online tests were conducted using a moving window approach, to generate a 'virtual' buffer of data to be given as input to AC-REG. Specifically, we analyzed how accuracy and computational time vary with different channel numbers, sampling frequencies and data buffer sizes. The number of EEG channels was equal to $16,32,64,128$ and 256 , respectively, and the sampling frequency was set to 200,500 and $1000 \mathrm{~Hz}$, respectively; different lengths of the buffer size were tested, in the range between 100 and $2000 \mathrm{~ms}$. Accuracy was quantified by using the correlation between the reconstructed EEG data and those without tACS artifact. Computation time was measured using MATLAB (release 2016b) running under MacOS (with $2.5 \mathrm{GHz}$ Intel Core i7 processor and 16 GB RAM). 


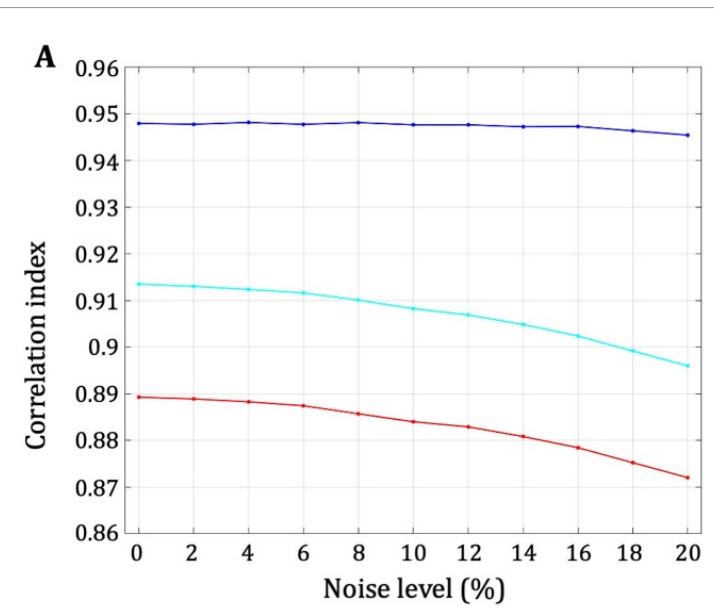

B

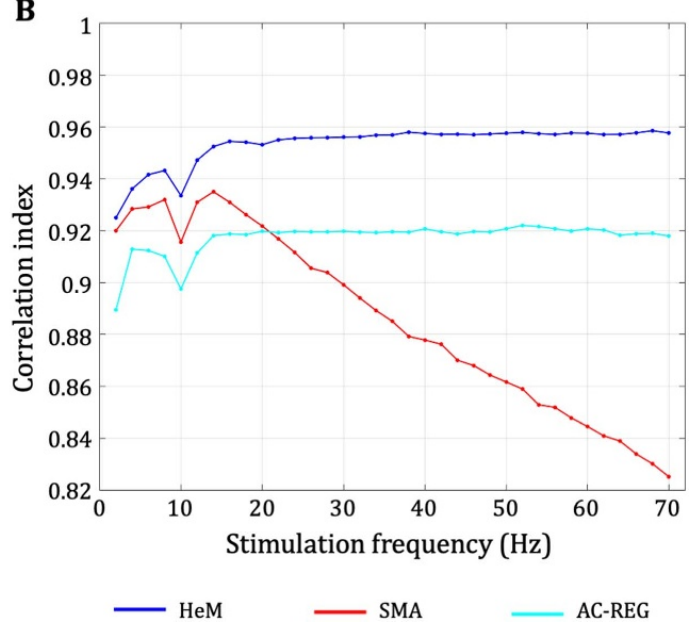

Figure 5. Performance of AC-REG, SMA and HeM for different noise levels and stimulation frequencies. Accuracy in the reconstruction of artifact-free EEG signals, as quantified by the correlation index, was examined (A) for noise levels varying between $0 \%$ and $20 \%$ of the signal intensity and (B) for stimulation frequency ranging between 1 and $70 \mathrm{~Hz}$.

Subsequently, we focused on EEG simulations with 64 channels and with sampling frequency at $500 \mathrm{~Hz}$. The size of the data buffer for AC-REG was set to $500 \mathrm{~ms}$. Hence, we compared the accuracy of AC-REG in tACS artifact attenuation with respect to two alternative approaches: SMA (Kohli and Casson 2015) and the Helfrich method (HeM) (Helfrich et al 2014). As for SMA, we used the implementation included in the ARtACS toolbox. Accordingly, the parameter $M$ was set equal to 10 , and the parameter $N$ was equal to the number of stimulation cycles. HeM was implemented in MATLAB, based on the information included in the original study. First, we assessed the sensitivity of AC-REG, SMA and HeM with respect to the noise level, which was varied between $0 \%$ and $20 \%$ of the average standard deviation of the clean EEG signal. Furthermore, after selecting a noise level of 5\% (Bai and $\mathrm{He}$ 2006), we compared the accuracy of the three methods as a function of the stimulation frequency.

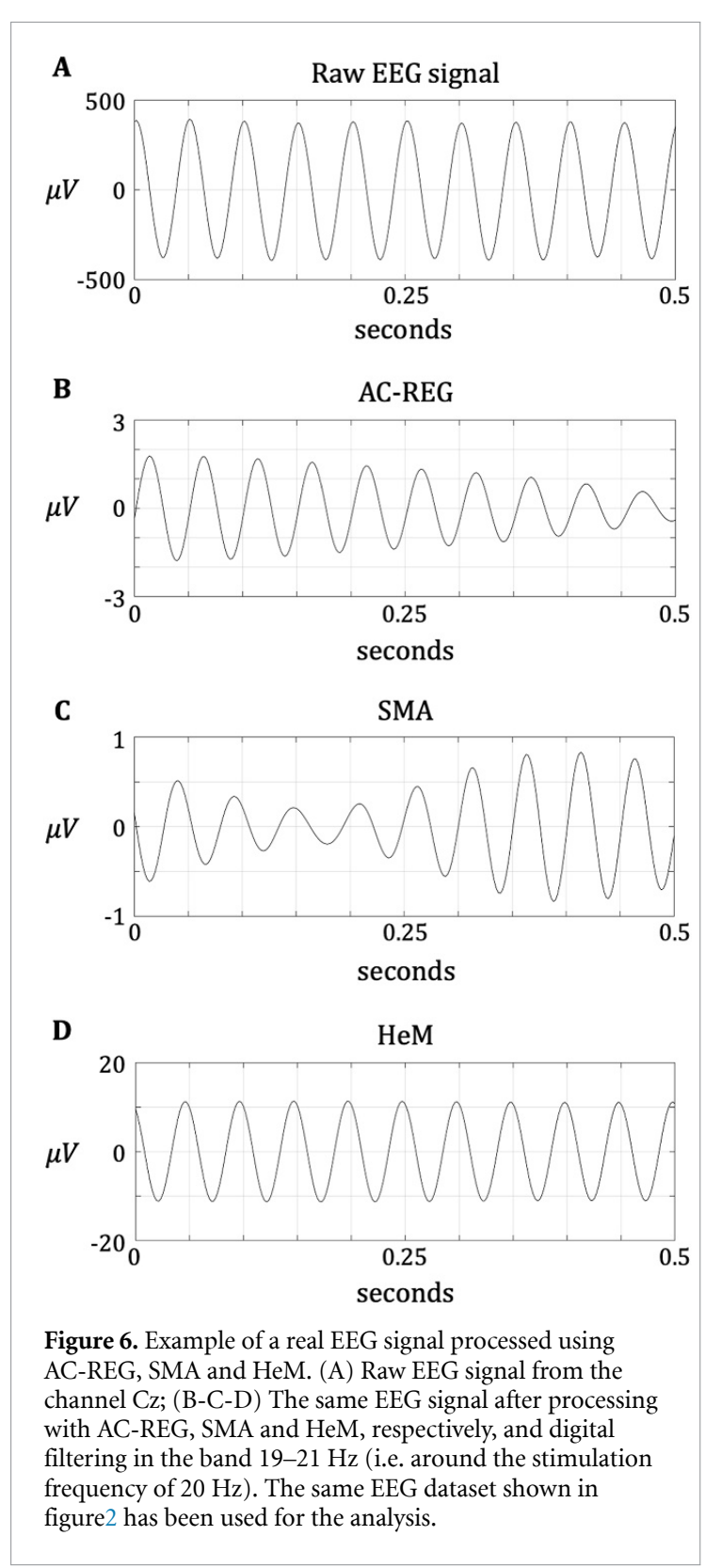

\subsubsection{Performance analysis using real EEG data}

We used recordings collected in 18 healthy righthanded volunteers (nine males and nine females, age ranging between 20 and 36). Participants gave their written consent before taking part in the experiment. The whole procedure was carried out in accordance with the principles of the Declaration of Helsinki, and the protocol was approved by the Local Ethical Committee of Chieti University.

tACS was delivered by a battery-driven, current stimulator (DC-Stimulator, NeuroConn GmbH, Germany) through a pair of conductive rubber electrodes $\left(3 \mathrm{~cm} \times 3 \mathrm{~cm}, 9 \mathrm{~cm}^{2}\right)$. An electroconductive gel was applied under the rubber electrodes to reduce contact impedance. We used a stimulation of $90 \mathrm{~s}$ duration and fade-in/fade-out times of $5 \mathrm{~s}$. A bilateral montage was used, with patch electrodes placed at the C3 and C4 sites of the 10-20 EEG system. During the tACS stimulation, an alternating 


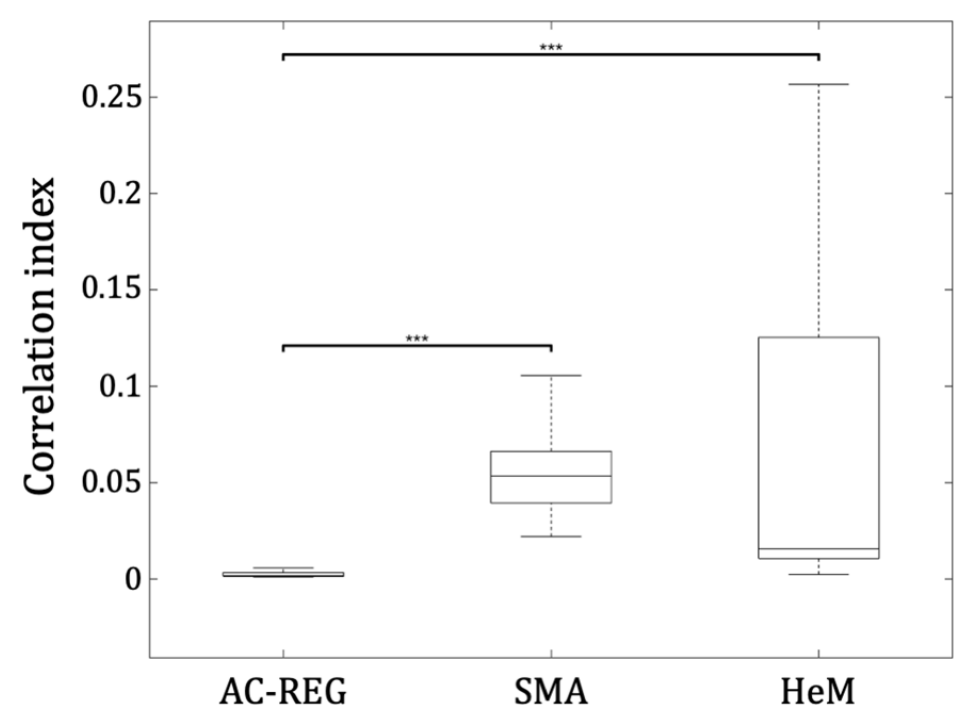

Figure 7. Quantification of tACS artifact residual in real EEG data. We estimated the correlation between EEG signals processed using AC-REG, SMA and HeM, filtered in the band 19-21 Hz, and the same signal before artifact correction. Correlations were averaged across channels to obtain a single value for each participant, and for each method. These values were displayed in the form of interquartile box plots. Statistical comparisons at the group level were performed using a Wilcoxon signed-rank test. Significance is indicated as follows: ${ }^{*}=\mathrm{p}<0.05,{ }^{* *}=\mathrm{p}<0.01,{ }^{* * *}=\mathrm{p}<0.001$.

A

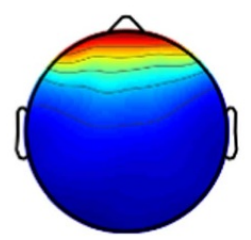

tACS off

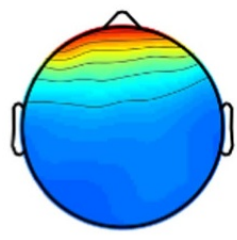

SMA
Ocular artifact component

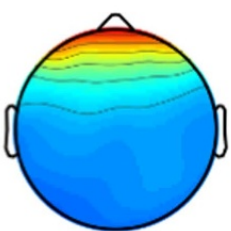

AC-REG

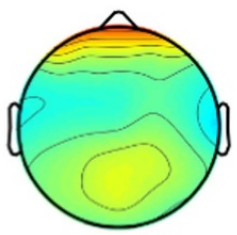

HeM
B

Occipital alpha component

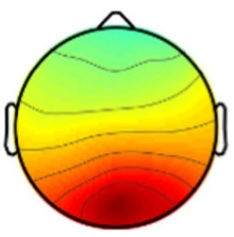

tACS off

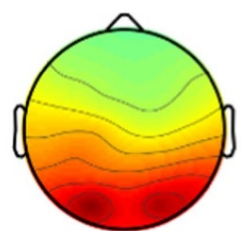

AC-REG
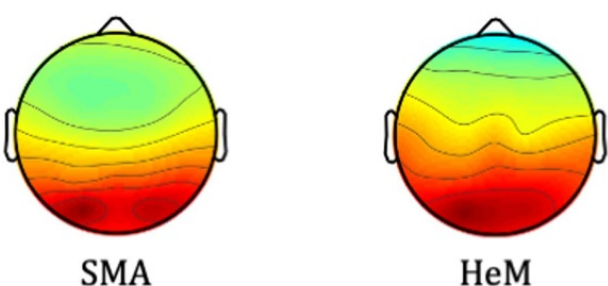
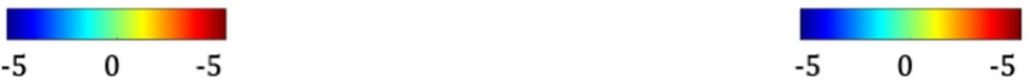

Figure 8. Topographies of ocular artifact and occipital alpha components in real EEG data with tACS off and on, respectively. Spatial distribution of (A) the ocular component, and (B) the occipital alpha component in a representative subject, for the tACS-off condition and the tACS-on condition after tACS artifact removal using AC-REG, SMA and HeM, respectively. The same EEG dataset shown in figure 2 has been used for the analysis.

current was transmitted with a sinusoidal waveform, the frequency was set at $20 \mathrm{~Hz}$ and the relative phase at $0^{\circ}$. Alternating current was applied at $1000 \mu \mathrm{A}$ resulting in a mean current density $0.011 \mathrm{~mA} \mathrm{~cm}^{-2}$.

EEG activity was recorded with a Be-plus system manufactured by Eb-Neuro (Florence, Italy) before, during and after tACS, using 57 scalp electrodes positioned according to a standard 10-10 montage. The electrode labeled as $\mathrm{AFz}$ was used as physical reference. Impedance of all electrodes was kept lower than $5 \mathrm{k} \Omega$. Signals were sampled at $512 \mathrm{~Hz}$ and stored on a computer for offline analysis. The EEG recording started $3 \mathrm{~min}$ before the stimulation and ended $3 \mathrm{~min}$ after it. The stimulation period lasted $90 \mathrm{~s}$. During the experiment, the participant was seated in a comfortable position and was asked to stay at rest,maintaining a relaxed position, with open eyes and without moving or engaging in any cognitive task. The EEG data were preprocessed offline, using the same analysis workflow used for the generation of the simulated data (see previous section). Specifically, bad-channel detection and interpolation, 

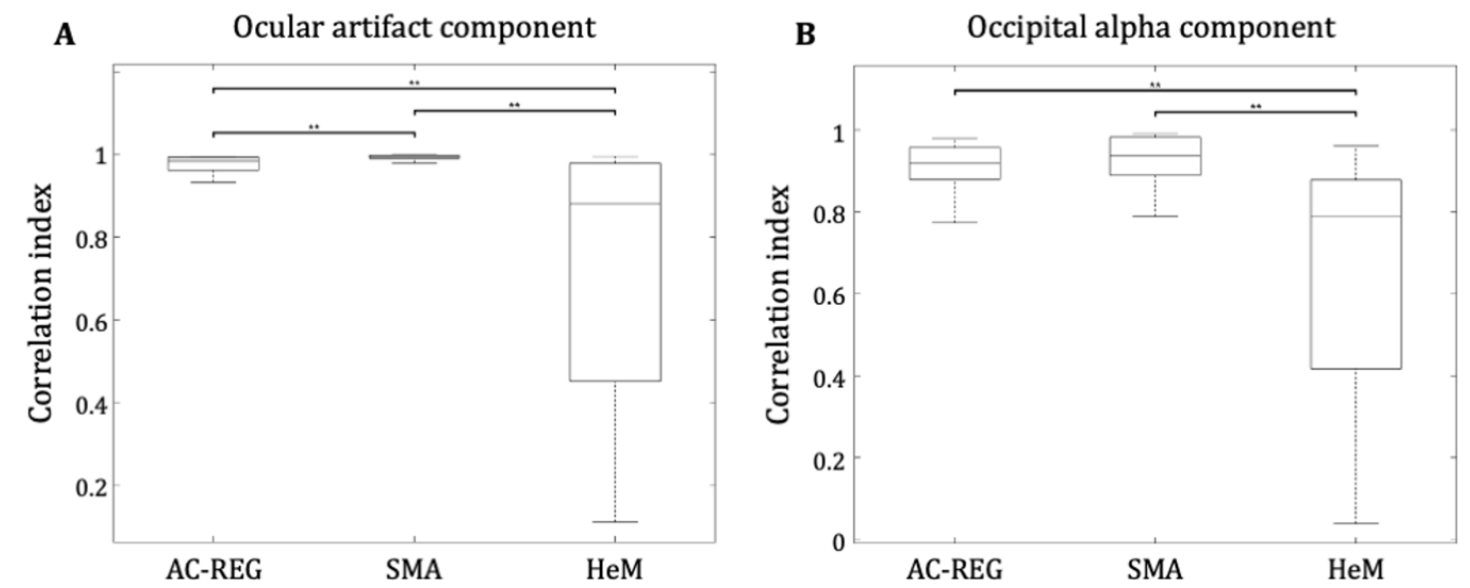

Figure 9. Quantification of ocular artifact and occipital alpha reconstruction in real EEG data. We estimated the impact of AC-REG, SMA and HeM processing, respectively, on the reconstruction of (A) the ocular artifact component and (B) the occipital alpha component. The components obtained from EEG data in the tACS-on condition, were compared with those obtained from EEG data in the tACS-off condition. Correlations were averaged across channels to obtain a single value for each participant, and for each method. These values were displayed in the form of interquartile box plots. Statistical comparisons at the group level were performed using a Wilcoxon signed-rank test. Significance is indicated as follows: ${ }^{*}=\mathrm{p}<0.05,{ }^{*} * \mathrm{p}<0.01,{ }^{* * *}=\mathrm{p}<0.001$.
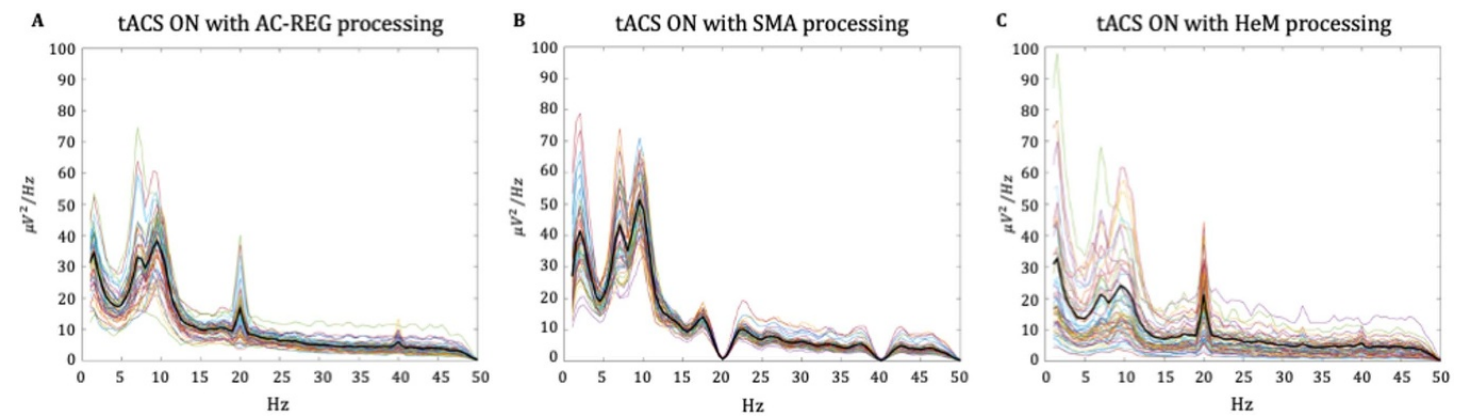

Figure 10. Effect of tACS artifact removal methods on spectral properties of EEG data. (A-C) PSDs from EEG data in the tACS-on period, processed using AC-REG, SMA and HeM, respectively. The same EEG dataset shown in figure 2 has been used for the analysis. The thick black line indicates the grand average of the PSD across channels.

digital filtering in the band $1-80 \mathrm{~Hz}$ and average rereferencing were applied (see figure 2 for an illustration of real EEG data).

To assess the effectiveness of AC-REG in attenuation the tACS artifact on real EEG data, we conducted three analyses. First, we quantified the correlation between the tACS signal and the artifact-corrected EEG data, filtered around the stimulation frequency $(19-21 \mathrm{~Hz})$. This analysis provided an indication concerning the presence of residual artifacts in the EEG data. SMA and HeM were used as benchmark to evaluate the performance of AC-REG. Second, we performed ICA of the EEG signals using the FastICA algorithm (Hyvarinen 1999), to extract specific components related to eye blinks and the occipital alpha rhythm. The topology of the components extracted from the artifact-free EEG data in the tACS-on period were compared to those from the EEG data in the tACS-off period, by means of their spatial correlation. Third, we investigated the correspondence in the power spectral density (PSD) of the artifact-free EEG data in tACS-on period as compared to the EEG data in the tACS-off period. This correspondence was quantified using the Pearson's correlation, focusing on all frequencies in the band $1-80 \mathrm{~Hz}$ except the stimulation frequency and its harmonics (i.e. 20, 40, $60 \mathrm{~Hz}$ ). A Wilcoxon signed rank test was used to test whether there were significant differences among the correlation values obtained with the three methods. The Bonferroni method was used to correct statistical significance, accounting for multiple comparisons (AC-REG vs. SMA, AC-REG vs. HeM, SMA vs. HeM). For all the analyses on real EEG data, AC-REG was used with a buffer size of $500 \mathrm{~ms}$.

\section{Results}

The performance of AC-REG depends on the amount of data that can be used for the definition of the spatial filter. To examine how the number of data channels, sampling frequencies and data buffer sizes impact on accuracy and computational time, we performed pseudo-online tests using the simulated EEG data. Overall, the accuracy in the reconstruction of 


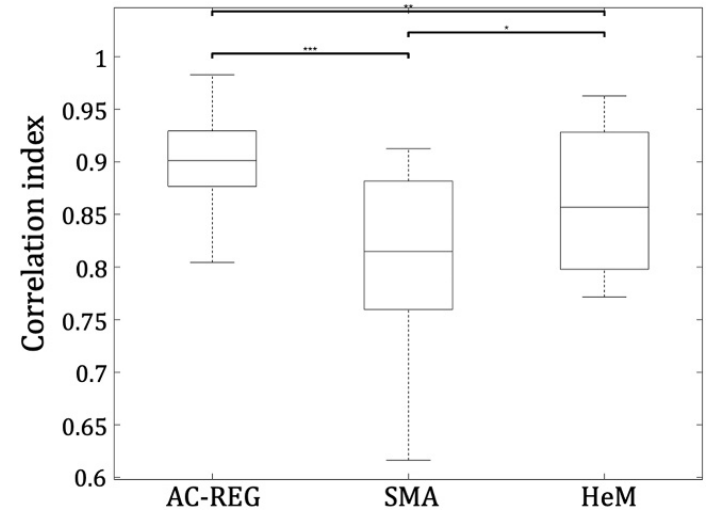

Figure 11. Performance of tACS artifact removal methods on real EEG data. We estimated the effectiveness of AC-REG, SMA and HeM in reconstructing artifact-free EEG signals. To this end, we used as reference the EEG data collected without stimulation. We quantified, channel by channel, the correlation between PSDs, and then we averaged the values across channels to obtain a single correlation value for each of the 18 participants, and for each method. The resulting correlation values were displayed in the form of interquartile box plots. Statistical comparisons at the group level were performed using a Wilcoxon signed-rank test. Significance is indicated as follows: ${ }^{*}=\mathrm{p}<0.05,{ }^{* *}=\mathrm{p}<0.01,{ }^{* * *}=\mathrm{p}<0.001$.

tACS artifact did not strongly depend on the number of EEG channels and on the sampling frequency, but better performance was nonetheless obtained with higher channel counts and higher sampling frequencies respectively (figure $3(\mathrm{~A})$ ). In contrast, the buffer size used for AC-REG processing was an important factor in determining the quality in tACS artifact reconstruction. Specifically, the use of a buffer size of at least $500 \mathrm{~ms}$ for AC-REG seemed to be important to ensure an effective artifact attenuation (figure $3(\mathrm{~B})$ ). We also found that the computation time had a super-linear relationship with channel count (figure 4(A)), whereas it increased with both sampling frequency and buffer size in a sub-linear manner (figure 4(B)).

By using simulated EEG data, we could also assess the performance of AC-REG with respect to alternative approaches, such as SMA and HeM. As expected, the correlation between simulated and reconstructed EEG data was only slightly dependent on the noise level for any method under investigation, possibly due to the high intensity of the tACS artifact. Notably, correlations were higher for AC-REG than SMA and HeM (figure 5(A)). Interestingly, we found that the performance of AC-REG, SMA and HeM depended on the tACS frequency in a substantial manner (figure 5(B)). All methods had a performance drop below $4 \mathrm{~Hz}$ and at around $10 \mathrm{~Hz}$, which are the frequencies with relatively lower ratio between artifact and neural signal amplitudes, respectively. Overall, we observed that SMA had a clear decrease in reconstruction accuracy with an increase of the stimulation frequency, whereas the performance of
AC-REG and HeM was largely stable across frequencies.

We finally moved to the assessment of AC-REG using real EEG data. First, we examined whether the EEG data processed by AC-REG, SMA and HeM possibly included residuals of the tACS signal. We found that the EEG signal at the stimulation frequency was in phase with the tACS artifact for HeM, whereas this was not the case for AC-REG and SMA (figure 6). The correlations between the tACS signal and the EEG signals at the stimulation frequency were significantly lower for AC-REG than SMA and HeM (both $\mathrm{p}<0.001$, with Bonferroni correction). Conversely, the correlation values obtained for SMA and HeM were not significantly different across participants ( $\mathrm{p}=0.557$, before Bonferroni correction) (figure 7).

Next, we extracted and analyzed the EEG components associated with eye movements and the occipital alpha rhythm, respectively (figure 8). ACREG, SMA and HeM permitted to reconstruct the ocular component and occipital alpha components. The EEG data processed with SMA had the highest correlations between the ocular componentestimated in the tACS-on and tACS-off periods, respectively (figure 9(A)). These correlations were significantly higher than those obtained ed with AC-REG and HeM ( $\mathrm{p}=0.002$ and $\mathrm{p}=0.0013$, respectively, with Bonferroni correction). Also, the values of ACREG were significantly higher than those of HeM ( $\mathrm{p}=0.018$, with Bonferroni correction). For the occipital alpha component, no significant differences were found between the correlations obtained with AC-REG and SMA processing, respectively ( $\mathrm{p}=0.5614$, before Bonferroni correction). In turn, the values of AC-REG and SMA were significantly higher than those obtained with HeM ( $p=0.003$ and $\mathrm{p}=0.006$, respectively, with Bonferroni correction).

The PSD of the EEG signals reconstructed using AC-REG clearly showed a single prominent peak at around $10 \mathrm{~Hz}$ and revealed two additional narrow peaks at the main stimulation frequency $(20 \mathrm{~Hz})$ and its first harmonic $(40 \mathrm{~Hz})$, respectively (figure 10). The same two peaks are easily detectable also in the PSD of the EEG signals reconstructed using HeM. With this method, however, the peak at around $10 \mathrm{~Hz}$ was much less prominent. In turn, the PSD obtained using SMA showed a clear peak at around $10 \mathrm{~Hz}$, whereas frequencies around 20 and $40 \mathrm{~Hz}$ were completely suppressed. In order to perform a more quantitative analysis, we used the PSD of the EEG data in the tACS-off condition as reference, and we quantified its correlation with the PSDs produced by AC-REG, SMA and HeM, respectively (figure 11). A Wilcoxon signed rank test showed that AC-REG had significantly higher values than SMA and HeM $(\mathrm{p}<0.001$ and $\mathrm{p}=0.0222$, respectively, with Bonferroni correction). Also, the values of SMA and HeM were not significantly different $(\mathrm{p}=0.075$, with Bonferroni correction). 


\section{Discussion}

The AC-REG method has been developed for attenuation of tACS artifacts in EEG signals. Due to its low-computational complexity, it may be effectively used in noninvasive closed-loop neuromodulation experiments. AC-REG uses an adaptive spatial filter based on PCA to dynamically estimate and subtract the tACS signal from EEG recordings. It outperforms alternative methods, both on simulated and real data. The low computation time may permit its use in online modality not only with low-density but also high-density EEG recordings. Importantly, the compatibility with high-density EEG may enable the use of AC-REG for source-level closed-loop neuromodulation (Guarnieri et al 2020).

\subsection{Primary features of AC-REG}

The AC-REG method relies on the use of PCA, a data decomposition technique ensuring low computation times required for noninvasive closed-loop experiments. Specifically, the identification of principal components temporally coherent with tACS signals permits to obtain a spatial filter to be used for artifact removal. By using a data buffer, the spatial filter is dynamically updated such that artifact-free EEG data can be obtained in an online manner. A possible caveat of our approach is that the data buffer should contain enough samples for an effective PCA decomposition, hence for an accurate definition of the spatial filter. On the other hand, it should be considered that computation times need to be kept as low as possible to enable the use of AC-REG in closed-loop neuromodulation experiments. Our results suggest that AC-REG can be used for the simultaneous processing of up to 256 EEG recordings, with low computation time. This is essential for the development of source-level closed-loop neuromodulation systems, in which neural activity in the brain is estimated and used for tuning brain stimulation parameters (Guarnieri et al 2020).

\subsection{Analysis of method performance}

To assess the performance of the AC-REG, we first relied on a simulated dataset containing a mix of neural signals and tACS artifacts. In this manner, we could define the buffer size of AC-REG yielding a reasonable compromise between accuracy and computation time (figures 3-4). With known ground truth, we could also examine the effectiveness of AC-REG in tACS artifact removal with alternative approaches, such as SMA and HeM, under controlled conditions. We found that AC-REG outperformed the other methods for all the noise levels and the stimulation frequencies that were tested (figure 5). Notably, AC-REG permitted the simultaneous processing of up to 256 channels (figure 4) with relatively low processing time, theoretically compatible with its use in quasi real-time applications. It should be considered that the processing of all EEG channels simultaneously can enable source localization in real-time (Guarnieri et al 2020).

The analysis conducted using AC-REG, SMA and HeM on real EEG data largely confirmed the findings from simulated data, and provided further insights in the capability of the methods for preserving true neural oscillations. The analysis of the artifact-corrected EEG data suggested that AC-REG successfully removes the sinusoidal signal that completely synchronized with the stimulation, possibly preserving neural signals entrained by the stimulation itself (figures 6-7). Notably, other sources of EEG activity were also preserved by AC-REG. For instance, we were able to reconstruct components associated with eye blinks and the occipital alpha rhythm (figure 8). In this regard, SMA seemed to be more accurate than AC-REG in preserving eye blinks, whereas no significant differences in reconstruction performance were found between AC-REG and SMA for the occipital alpha rhythm (figure 9). To evaluate the performance of AC-REG, SMA and $\mathrm{HeM}$ on real data, we also compared the spectral properties of EEG signals in tACS-on and tACS-off conditions. It should be noted that modulations of neural activity and connectivity during and following tACS have been reported (Neuling et al 2013, Vosskuhl et al 2016, Bächinger et al 2017). Considering the relatively short duration of the stimulation and the strength of the injected current, any assessment of stimulation-induced neural changes needs to be conducted with caution (Vöröslakos et al 2018). On the other hand in this study, we focused on gross alterations in power spectrum density introduced by artifact removal methods. Our results suggested that AC-REG could largely preserve EEG signals for frequencies below the one of the stimulation itself (figures 10-11). We also found that, unlike ACREG and HeM, SMA strongly suppressed all the harmonics associated with the tACS artifact, inducing distortions in the frequency content of neural signals. The differences between AC-REG and HeM were less marked, although still significant. Notably, the use of $\mathrm{HeM}$ yielded an overall reduction of power at all frequencies compared to AC-REG and SMA, in particular at around $10 \mathrm{~Hz}$.

\subsection{Limitations and future work}

In this study, we introduced AC-REG and validated it using both simulated and real EEG data. Simulated EEG data were created by adding sinusoidal signals generated using the ARtACS toolbox to artifactcleaned EEG signals. Temporal distortions introduced by changes in electrode impedance occurring during data acquisition were modelled using an OrnsteinUhlenbeck process. However, the possible impact of time-sample jitters (Barban et al 2019) and biological artifacts (Noury and Siegel 2017) was not taken into 
account. The validation on real data was conducted by testing the correspondence between the PSDs with tACS on and off, respectively, in resting state condition. This approach can be justified by the fact that the stimulation induces entrainment primarily of brain oscillations at the frequency of the tACS and its harmonics (Antal and Herrmann 2016, Adaikkan and Tsai 2020). To ensure the generalizability of our results, it would be important to extend the validation of AC-REG on real EEG data collected with other experimental protocols. In particular, event-related protocols may be used to test whether the reconstruction of neural activity at the single-trial level is unaffected by residual noise (Giroldini et al 2016, Mantini et al 2007, Helfrich et al 2014). Another important aspect to consider is that real EEG data were collected during tACS at $20 \mathrm{~Hz}$. It is therefore worth investigating how our findings might generalize to other stimulation frequencies, also considering that non-linear distortions can be more easily observed at higher stimulation frequencies (Noury et al 2016).

AC-REG was introduced and validated using methods typically used for offline analyses, such as SMA and HeM. The comparison may be extended in future studies to other methods that have been proposed to attenuate high-voltage simulation artifacts mixed in EEG recordings, as for instance quadratureregression independent vector analysis (q-IVA) (Lee et al 2019). This method has been so far validated only on EEG data collected during galvanic vestibular stimulation, but it could be readily used to attenuate artifacts generated by tACS. AC-REG may be particularly valuable for applications in which EEG recordings need to be processed simultaneously and in real-time, as for instance in source-based closed loop experiments (Bergmann et al 2016). It should be noted, however, that the specific multi-channel implementation of AC-REG make the method potentially sensitive to the presence of bad channels. In this regard, specific solutions for enabling the effective use of AC-REG in online modality will need to be addressed in future studies. It would be important to test the effectiveness and validity of AC-REG in a noninvasive closed-loop neuromodulation experiment. As such, EEG data will have to be processed in real-time for the attenuation of the tACS artifact (Schlegelmilch et al 2013, Kohli et al 2017), as well as biological signals (Guarnieri et al 2018). The readout of EEG activity will then be used to adjust phase and intensity of the stimulation (Bergmann et al 2016).

\section{Conclusions}

We have introduced AC-REG, a method for the attenuation of tACS artifacts removal that is suitable not only for low-density but also high-density EEG recordings. The method requires the use of PCA on short time windows for the dynamic update of a spatial filter. This ensures low computation times, potentially enabling its use in real-time during EEG experiments. Just as importantly, AC-REG outperforms other methods for tACS artifact removal, which normally operate in an offline modality. We argue that AC-REG may enable further development of closedloop neuromodulation techniques, with potential applications both in healthy individuals and in neurological patients.

\section{Acknowledgments}

The work was supported by the KU Leuven Special Research Fund (Grant No. C16/15/070), the Research Foundation Flanders (FWO) (Grant Nos. G0F76.16N, G0936.16N and EOS.30446199, and $\mathrm{PhD}$ fellowship $1104520 \mathrm{~N}$ to $\mathrm{RG}$ ) and the Italian Ministry of Health (Grant No. RF-2018-12366899).

\section{ORCID iDs}

Roberto Guarnieri (1) https://orcid.org/0000-00015019-2897

Alfredo Brancucci () https://orcid.org/0000-00026826-1341

Anita D’Anselmo (1) https://orcid.org/0000-00033940-2616

Valerio Manippa (ㅁ) https://orcid.org/0000-00033892-5212

Stephan P Swinnen (1) https://orcid.org/0000-00017173-435X

Franca Tecchio (1) https://orcid.org/0000-0002-13255059

Dante Mantini (1) https://orcid.org/0000-0001-64855559

\section{References}

Adaikkan C and Tsai L-H 2020 Gamma entrainment: impact on neurocircuits, glia, and therapeutic opportunities Trends Neurosci. 43 24-41

Alekseichuk I, Falchier A Y, Linn G, Xu T, Milham M P, Schroeder C E and Opitz A 2019 Electric field dynamics in the brain during multi-electrode transcranial electric stimulation Nat. Commun. 102573

Alexander M L, Alagapan S, Lugo C E, Mellin J M, Lustenberger C, Rubinow D R and Fröhlich F 2019 Double-blind, randomized pilot clinical trial targeting alpha oscillations with transcranial alternating current stimulation (tACS) for the treatment of major depressive disorder (MDD) Transl. Psychiatry 9106

Ali M M, Sellers K K and Fröhlich F 2013 Transcranial alternating current stimulation modulates large-scale cortical network activity by network resonance J. Neurosci. 33 11262-75

Antal A and Herrmann C S 2016 Transcranial alternating current and random noise stimulation: possible mechanisms Neural Plast. 20163616807

Bai X and He B 2006 Estimation of number of independent brain electric sources from the scalp EEGs IEEE Trans. Biomed. Eng. 53 1883-92

Barban F, Buccelli S, Mantini D, Chiappalone M and Semprini M 2019 Removal of tACS artefact: A simulation study for 
algorithm comparison 9th Int. IEEE/EMBS Conf. on Neural Engineering (NER) pp 393-6

Beres A M 2017 Time is of the essence: a review of electroencephalography (EEG) and event-related brain potentials (ERPs) in language research Appl. Psychophysiol. Biofeedback 42 247-55

Bergmann T O, Karabanov A, Hartwigsen G, Thielscher A and Siebner H R 2016 Combining non-invasive transcranial brain stimulation with neuroimaging and electrophysiology: current approaches and future perspectives Neuroimage 140 4-19

Bächinger $\mathrm{M}$ et al 2017 Concurrent tACS-fMRI reveals causal influence of power synchronized neural activity on resting state fMRI connectivity J. Neurosci. 37 4766-77

Cabral-Calderin Y, Williams K A, Opitz A, Dechent P and Wilke M 2016 Transcranial alternating current stimulation modulates spontaneous low frequency fluctuations as measured with fMRI NeuroImage 141 88-107

Cappon D, D’Ostilio K, Garraux G, Rothwell J and Bisiacchi P 2016 Effects of $10 \mathrm{~Hz}$ and $20 \mathrm{~Hz}$ transcranial alternating current stimulation on automatic motor control Brain Stimulation 9 518-24

Clayton M S, Yeung N and Cohen Kadosh R 2018 The effects of 10 $\mathrm{Hz}$ transcranial alternating current stimulation on audiovisual task switching Front. Neurosci. 1267

Dowsett J, Herrmann C and Taylor P 2019 Modulation of SSVEPs using frequency matched tACS Brain Stimulation 12418

Feurra M, Bianco G, Santarnecchi E, Del Testa M, Rossi A and Rossi S 2011 Frequency-dependent tuning of the human motor system induced by transcranial oscillatory potentials I. Neurosci. 31 12165-70

Giroldini W, Pederzoli L, Bilucaglia M, Melloni S and Tressoldi P 2016 A new method to detect event-related potentials based on Pearson's correlation EURASIP J. Bioinform. Syst. Biol. 201611

Golub G and Kahan W 1965 Calculating the singular values and pseudo-inverse of a matrix J. Soc. Ind. Appl. Math. B 2 205-24

Grossman N et al 2017 Noninvasive deep brain stimulation via temporally interfering electric fields Cell 169 1029-1041.e16

Guarnieri R, Marino M, Barban F, Ganzetti M and Mantini D 2018 Online EEG artifact removal for BCI applications by adaptive spatial filtering J. Neural Eng. 15056009

Guarnieri R, Zhao M, Taberna G A, Ganzetti M, Swinnen S P and Mantini D 2020 RT-NET: real-time reconstruction of neura activity using high-density electroencephalography Neuroinform. https://doi.org/10.1007/s12021-020-09479-3

Guggenmos D J, Azin M, Barbay S, Mahnken J D, Dunham C, Mohseni P and Nudo R J 2013 Restoration of function after brain damage using a neural prosthesis Proc. Natl Acad. Sci. USA 110 21177-82

Helfrich R F, Schneider T R, Rach S, Trautmann-Lengsfeld S A, Engel A K and Herrmann C S 2014 Entrainment of brain oscillations by transcranial alternating current stimulation Curr. Biol. 24 333-9

Herrmann C S, Rach S, Neuling T and Strüber D 2013 Transcranial alternating current stimulation: A review of the underlying mechanisms and modulation of cognitive processes Front. Hum. Neurosci. 7279

Hyvarinen A 1999 Fast and robust fixed-point algorithms for independent component analysis IEEE Trans. Neural Networks 10 626-34

Iturrate I, Pereira M and Millán J D R 2018 Closed-loop electrica neurostimulation: challenges and opportunities Curr. Opin. Biomed. Eng. 8 28-37

Kohli S and Casson A J 2020 Machine learning validation of EEG+tACS artefact removal J. Neural Eng. 1716034

Kohli S and Casson A J 2019 Removal of gross artifacts of transcranial alternating current stimulation in simultaneous EEG monitoring Sensors 19190

Kohli S and Casson A J 2015 Removal of transcranial a.c. current stimulation artifact from simultaneous EEG recordings by superposition of moving averages Proc. of the Annual Int Conf. of the IEEE Eng. Med. Biol. Soc. pp 3436-9

Kohli S, Krachunov S and Casson A J 2017 Towards closed-loop transcranial electrical stimulation: a comparison of methods for real time tES-EEG artefact removal using a phantom head model Brain Stimulation $10467-8$

Lee S, Mckeown M J, Wang Z J and Chen X 2019 Removal of high-voltage brain stimulation artifacts from simultaneous EEG recordings IEEE Trans. Biomed. Eng. 66 50-60

Liu Q, Farahibozorg S, Porcaro C, Wenderoth N and Mantini D 2017 Detecting large-scale networks in the human brain using high-density electroencephalography Hum. Brain Mapp. 38 4631-43

Mansouri F, Dunlop K, Giacobbe P, Downar J and Zariffa J 2017 A fast EEG forecasting algorithm for phase-locked transcranial electrical stimulation of the human brain Front. Neurosci. 11 1-14

Mantini D, Perrucci M G, Cugini S, Ferretti A, Romani G L and Del Gratta C 2007 Complete artifact removal for EEG recorded during continuous fMRI using independent component analysis NeuroImage 34 598-607

Marshall L, Kirov R, Brade J, Mölle M and Born J 2011 Transcranial electrical currents to probe EEG brain rhythms and memory consolidation during sleep in humans PLoS One 6 e12905

Merlet I et al 2013 From oscillatory transcranial current stimulation to scalp EEG changes: a biophysical and physiological modeling study PLoS One 8 e57330

Morales-Quezada L, Cosmo C, Carvalho S, Leite J, Castillo-Saavedra L, Rozisky J R and Fregni F 2014 Cognitive effects and autonomic responses to transcranial pulsed current stimulation Exp. Brain Res. 233 701-9

Neuling T, Rach S and Herrmann C 2013 Orchestrating neuronal networks: sustained after-effects of transcranial alternating current stimulation depend upon brain states Front. Hum. Neurosci. 7161

Noury N, Hipp J F and Siegel M 2016 Physiological processes non-linearly affect electrophysiological recordings during transcranial electric stimulation NeuroImage 140 99-109

Noury N and Siegel M 2017 Phase properties of transcrania electrical stimulation artifacts in electrophysiological recordings NeuroImage 158 406-16

Pahor A and Jaušovec N 2018 The effects of theta and gamma tacs on working memory and electrophysiology Front. Hum. Neurosci. 11651

Perlmutter J S and Mink J W 2006 Deep brain stimulation Annu. Rev. Neurosci. 29 229-57

Rebesco J M, Stevenson I H, Körding K P, Solla S A and Miller L E 2010 Rewiring neural interactions by micro-stimulation Front. Syst. Neurosci. 439

Riecke L, Formisano E, Herrmann C S and Sack A T 2015 4-Hz transcranial alternating current stimulation phase modulates hearing Brain Stimulation 8 777-83

Samogin J, Liu Q, Marino M, Wenderoth N and Mantini D 2019 Shared and connection-specific intrinsic interactions in the default mode network NeuroImage 200 474-81

Schlegelmilch F, Schellhorn K and Stein P 2013 A method for online correction of artifacts in EEG signals during transcranial electrical stimulation Clin. Neurophysio 124 e166-8

Semprini M, Laffranchi M, Sanguineti V, Avanzino L, De Icco R, De Michieli L and Chiappalone M 2018 Technological approaches for neurorehabilitation: from robotic devices to brain stimulation and beyond Front. Neurol. 9212

Sugata $\mathrm{H}$ et al 2018 Modulation of motor learning capacity by transcranial alternating current stimulation Neuroscience 391 131-9

Sun F T and Morrell M J 2014 Closed-loop neurostimulation: the clinical experience Neurotherapeutics 11 553-63

Tucker D M 1993 Spatial sampling of head electrical fields: the geodesic sensor net Electroencephalogr. Clin. Neurophysiol. 87 154-63 
Turnip A and Junaidi E 2014 Removal artifacts from EEG signal using independent component analysis and principal component analysis 2014 2nd Int. Conf. on Technology, Informatics, Management, Engineering \& Environment pp 296-302

Vossen A, Gross J and Thut G 2015 Alpha power increase after transcranial alternating current stimulation at alpha frequency (a-tACS) reflects plastic changes rather than entrainment Brain Stimulation 8 499-508
Vosskuhl J, Huster R J and Herrmann C S 2016 BOLD signal effects of transcranial alternating current stimulation (tACS) in the alpha range: A concurrent tACS-fMRI study NeuroImage 140 118-25

Vöröslakos M et al 2018 Direct effects of transcranial electric stimulation on brain circuits in rats and humans Nat. Commun. 9483

Woods A J et al 2016 A technical guide to tDCS, and related non-invasive brain stimulation tools Clin. Neurophysiol 127 1031-48 